\title{
Nano-therapeutic cancer immunotherapy using hyperthermia-induced heat shock proteins: insights from mathematical modeling
}

This article was published in the following Dove Press journal:

International Journal of Nanomedicine

\author{
Fang-Chu Lin \\ Chao-Hsiung Hsu \\ Yung-Ya Lin \\ Department of Chemistry and \\ Biochemistry, University of California, \\ Los Angeles, CA, USA
}

Background: Nano-therapeutic utilizing hyperthermia therapy in combination with chemotherapy, surgery, and radiation is known to treat various types of cancer. These cancer treatments normally focus on reducing tumor burden. Nevertheless, it is still challenging to confine adequate thermal energy in a tumor and obtain a complete tumor ablation to avoid recurrence and metastasis while leaving normal tissues unaffected. Consequently, it is critical to attain an alternative tumor-killing mechanism to circumvent these challenges. Studies have demonstrated that extracellular heat shock proteins (HSPs) activate antitumor immunity during tumor cell necrosis. Such induced immunity was further shown to assist in regressing tumor and reducing recurrence and metastasis. However, only a narrow range of thermal dose is reported to be able to acquire the optimal antitumor immune outcome. Consequently, it is crucial to understand how extracellular HSPs are generated.

Materials and methods: In this work, a predictive model integrating HSP synthesis mechanism and cell death model is proposed to elucidate the HSP involvement in hyperthermia cancer immune therapy and its relation with dead tumor cells. This new model aims to provide insights into the thermally released extracellular HSPs by dead tumor cells for a more extensive set of conditions, including various temperatures and heating duration time.

Results: Our model is capable of predicting the optimal thermal parameters to generate maximum HSPs for stimulating antitumor immunity, promoting tumor regression, and reducing metastasis. The obtained nonlinear relation between extracellular HSP concentration and increased dead cell number, along with rising temperature, shows that only a narrow range of thermal dose is able to generate the optimal antitumor immune result.

Conclusion: Our predictive model is capable of predicting the optimal temperature and exposure time to generate HSPs involved in the antitumor immune activation, with a goal to promote tumor regression and reduce metastasis.

Keywords: nano-therapeutic, nanomedicine, hyperthermia, heat shock protein, cancer immunotherapy

\section{Introduction}

The combination of nanomedicine and hyperthermia for cancer treatment is receiving increased attention. For example, it was shown that, by utilizing gold nanorods and laser irradiation as an external heating source, hyperthermia in combination with chemotherapy led to more than $90 \%$ of cell inviability. ${ }^{1}$ Alternating magnetic field hyperthermia for cancer treatment has also been shown to play an effective therapeutic role due to its ability to selectively target the cells of interest and induce local heating while minimizing secondary effects in surrounding healthy tissues. ${ }^{2}$ Drug delivery
Correspondence: Yung-Ya Lin Department of Chemistry and Biochemistry, University of California, Los Angeles, CA 90095, USA

Tel +I 3I0 2062856

Fax + I 310 2064038

Email yylin@chem.ucla.edu 
systems incorporated with magnetic nanoparticles can also take advantage of induced heating under alternating magnetic fields to perform desired drug release. ${ }^{2}$ A much improved tumor ablation after alternating magnetic field hyperthermia was achieved when intravenous injection of magnetic nanoparticles was administrated. ${ }^{3}$ Also, methotrexate-coupled magnetic nanoparticle using combined treatment with hyperthermia was employed, aiming to bypass severe side effects in nontarget organs resulting from the heterogeneous tumor cell responses. ${ }^{4}$ These hyperthermia studies mainly focused on directly killing tumor cells. Operating accurate tumor ablation to achieve complete tumor elimination and thus avoid metastasis, however, is still problematic. Consequently, to circumvent these challenges, attaining an alternative tumorkilling mechanism, such us hyperthermia therapy-activated immunomodulation, is critical in cancer treatment. ${ }^{5}$

There is a rapidly growing interest in the combination of immunotherapy with hyperthermia for cancer treatment. Primary tumors treated with hyperthermia have led to spontaneous regression of untreated, distant metastases due to the heat-induced antitumor immune response. ${ }^{5}$ It was demonstrated that local hyperthermia treatment applied to identified tumors in inducing antitumor immune responses activated by extracellular heat shock proteins (HSPs) potentially reduces the risk of recurrence and metastasis. ${ }^{6}$ Also, the level of HSPs in the sera of patients has been shown to be significantly increased after alternating magnetic field hyperthermia, where the increased serum levels of HSPs resulted in a better survival rate. ${ }^{7}$

HSPs are a family of proteins that are produced by cells in response to exposure to stressful conditions, including exposure to heat, cold, and UV light. HSPs are named according to their molecular weight. For example, the 70 kilodalton HSPs (HSP70s) are a family of conserved ubiquitously expressed HSPs, existing in virtually all living organisms. The HSP70s are an important part of the cell's machinery for protein folding and help to protect cells from stress. In this study, we will focus on HSP70 due to its immunogenic properties in the extracellular environment ${ }^{8}$ as well as for the comparison of our proposed model with the reported experimental data. ${ }^{9}$ As a result, we will denote HSP70 simply by HSP from now on. Studies have shown that hyperthermia-induced necrotic cell death correlated with HSP release. ${ }^{10}$ HSPs secreted into the extracellular space during cell necrosis act as danger signals and trigger adaptive antitumor immune mechanism. Tumor undergoing heat stress by hyperthermia was followed by the release of tumor antigens, which would be chaperoned by extracellular HSPs released from necrotic tumor cells.
The HSP tumor-antigen complexes facilitate the presentation of tumor antigens, induce the antitumor immunity, and activate immune cells. ${ }^{11}$ Many of HSP-antigen complexes bind to and activate antigen-presenting cells (APCs). ${ }^{12}$ Once APCs phagocytose HSP-antigen complex, APCs can present the antigens to cytotoxic $\mathrm{T}$ cells and initiate adaptive immune responses. ${ }^{13}$ The interaction of HSP with APCs has driven the development of HSP-containing vaccines. Vaccines designed to deliver antigens directly to dendritic cells have been shown to generate impressive immune responses and protection from tumor challenge. ${ }^{14} \mathrm{HSP}$-containing nanovaccines based on superparamagnetic iron oxide nanoparticles have demonstrated superior improvement in tumor targeting due to overexpression of HSP receptors on glioma and boost-up of antitumor responses. ${ }^{15,16}$ As a result, HSP can aid in tumor targeting and antitumor immune activation.

It is crucial to understand how temperature influences the immune system. Heat-induced cell necrosis is often mediated by externally applied thermal stress, which also elicits an upregulation of HSPs to protect cancer cells that undergo heat stress. ${ }^{17,18}$ Moderate hyperthermia with temperature $\leq 43^{\circ} \mathrm{C}$ was reported to promote thermotolerance, the ability possessed by intracellular HSPs to tolerate future hyperthermia treatments, by inducing HSP expression in cells. ${ }^{19}$ As a result, the applicability of the hyperthermia treatment may be initially limited due to thermotolerance resulted from the upregulation of intracellular HSP, ${ }^{20}$ which also assists in inhibiting apoptosis by protecting against cell injury..$^{5}$ Study showed that HSP release starts at $41^{\circ} \mathrm{C}$, reaches a maximum at $43^{\circ} \mathrm{C}$, and is completely diminished at $45^{\circ} \mathrm{C} .{ }^{9}$ Hyperthermia at a temperature above $43^{\circ} \mathrm{C}$ triggers protein denaturation, which leads to cellular death. ${ }^{21}$ Also, it was observed that when primary tumors were heated at $45^{\circ} \mathrm{C}$ instead of $43^{\circ} \mathrm{C}$, the resistance against secondary tumors was not established. ${ }^{13}$ On account of these results, we expect to see an increase in HSP synthesis when an elevated temperature of around $41^{\circ} \mathrm{C}$ is applied. Such increase is mainly contributed by the intracellular HSP induction. The production of HSP reaches the maximum at around $43^{\circ} \mathrm{C}$ due to the release of extracellular HSPs and then decrease when the temperature is further raised. Regarding heating exposure time, studies showed that irreversible cell damage only occurs after prolonged heat exposure when cell lines or tissues are exposed to temperatures of around $40^{\circ} \mathrm{C}-50^{\circ} \mathrm{C} .{ }^{5}$ Heating cells or tissues to temperatures $\geq 42^{\circ} \mathrm{C}$ for 1 or more hours could result in significant cell killing. ${ }^{22}$ This result can serve as a predicted approximate exposure time associated with the optimal temperature to achieve maximum extracellular 
HSP generation. Moreover, HSPs are thought to secrete into the extracellular space during cell necrosis, whereas it was observed that the secretion still takes place in the absence of cell death. ${ }^{23}$ For this reason, the existence of a secretory pathway was proposed..$^{23}$

This study investigated the secretion and synthesis of extracellular HSPs that are involved in antitumor immunological processes and their relation with necrotic cells. Because only extracellular HSPs participate in immune activation, the study will focus on the extracellular HSPs released from the necrotic cells after the cytotoxic temperature is achieved. Along these lines, we propose a new model that is capable of considering the amount of extracellular HSPs by incorporating HSP synthesis with cell death. Moreover, it has been reported that there seems to be a narrow range of optimal temperature for the heated tumor to induce adaptive immune responses. ${ }^{13}$ Such temperature dependence indicates that different temperatures provide a different level of impacts on the induced immune responses and highlights the need to understand temperature effects across a relatively narrow range of thermal dose. Thus, the proposed model will give a closer analysis on thermal parameters to address the issue of temperature sensitivity in stimulating antitumor immunity. The model parameters will be analyzed to find the heating temperature and the exposure time that optimize the release of extracellular HSPs, which are correlated with cell death under thermal stress and bring out the greatest antitumor immune outcome.

\section{Materials and methods Induction and generation of HSPs}

It is essential to consider the effect on the cellular responses, such as the upregulation of HSPs that occurs under heat stress due to thermotolerance effect, with a dynamic analysis. To account for such HSP-induced thermotolerance, which is triggered by the heat-shock response (HSR), a mathematical model of HSP-based HSR mechanism is introduced. ${ }^{13,24,25}$ It can be seen from the mathematical model that HSP is the main component of this hyperthermia-involved mechanism. The model depicts the induced dynamical HSP synthesis by an external temperature stimulus and its interactions with key intracellular components in the HSR mechanism. In this work, the model used to calculate HSP synthesis induced at elevated temperatures is constituted by 12 reactions, where one of the rate constants shows the temperature dependence of the whole model. ${ }^{24,25}$ The temperature-dependent rate constant describes the fraction of protein denaturation (native proteins $\stackrel{k_{11}^{T}}{\longrightarrow}$ denatured proteins) as a function of temperature and is written as

$$
k_{11}^{T} \approx m_{11} \times\left(1-\frac{0.4}{e^{T-37}}\right) \times 1.4^{T-37} \min ^{-1} T \epsilon[37,45]^{\circ} \mathrm{C}
$$

where $m_{11}$ is a time-scale dependent multiplier - a variable used to fit the experimental data in the right unit. $k_{11}^{T}$ was approximated from the experimental data of differential scanning calorimetry ${ }^{26,27}$ and is proportional to the function that describes the response of substrate (ie, the denatured protein) to the temperature.

\section{HSP synthesis and cell death model integration}

It was suggested that HSP induction and the initiation of thermotolerance are due to the HSP's protective tendency when they are exposed to stressors ${ }^{8}$ as well as the significant injury or dysfunction of some targeted tissues. ${ }^{28,29}$ Also, the improved thermotolerance of the feeblest organ and cell systems further gives rise to the thermotolerance development. ${ }^{30}$ Studies showed that heat stress that is involved with widespread cellular injury is relevant to the cell necrosis, while cell surface HSPs or those that are released from necrotic cells into extracellular environment participate in cellular antitumor immune activation. ${ }^{11,30}$ Therefore, to take the number of free extracellular HSPs as the index of the antitumor immune activation, the previously discussed HSR mechanism model is proposed to be integrated with a cell death model. This integration is based on an assumption that the number of antigen is in excess such that all extracellular HSPs can form complex with tumor antigens. In this work, we only look at the immunomodulatory activities exerted by the free HSP at extracellular localization that are released from the heat-induced dead cells without considering the activation of immune cells due to the membrane HSP expression. ${ }^{31}$

In this study, a three-state cell death model proposed by O'Neill et al ${ }^{32}$ was used to approximately determine the number of dead tumor cells in terms of heating temperature and exposure time. This model was chosen over Arrheniusbased models because it deals with continuous dynamic temperatures instead of a discrete, single temperature heating that would create a marked discontinuity at temperatures around $43^{\circ} \mathrm{C}$. The model contains three compartments: fully alive (A), fully dead (D), and vulnerable (V) compartments. These three compartments relate to each other by the following equation:

$$
\mathrm{A} \underset{k_{b}}{\stackrel{k_{f}}{\rightleftarrows}} \stackrel{k_{f}}{\rightarrow} \mathrm{D}
$$


The transition from fully alive (A) to vulnerable (V), ie, an injured state, is represented by a forward rate constant, $k_{f}$, while a backward rate constant, $k_{b}$, represents a selfhealing process from vulnerable (V) to fully alive (A) state. The simplification made in the three-state cell death model, where the transition from vulnerable (V) to fully dead (D) possesses the same forward rate constant, $k_{f}$, is based on an assumption that there is just a single damage process that accounts for all physiological damages, and thus, both transitions lead to the same result. The forward rate constant in this cell death model is defined as, ${ }^{32}$

$$
k_{f}=\overline{k_{f}} e^{\frac{T}{T_{k}}}(1-A),
$$

where $\overline{k_{f}}$ is a scaling constant and $T_{k}\left({ }^{\circ} \mathrm{C}\right)$ was set to let the rate of the exponential increase with temperature. The exponential curve accounts for the insignificant thermal damage at body temperature, and the fact that the alreadydamaged tissue is more prone to further damage. ${ }^{32}$ Also, the inclusion of $(1-A)$ considers the nonlinear influence from the damaged state of surrounding cells on the reaction dynamics. Therefore, the three-state cell death model allows the fitting of data over a wider thermotherapeutic temperature range, including the beginning phase when cells are first exposed to the heat shock.

Heating duration time, $\tau$, and the temperature, $T$, are the two experimental parameters that are often applied in the basic experimental practices. The free extracellular HSP concentration generation rate, $S(\tau, T)$, is the product of the HSP concentration and the number of dead cells per unit of time:

$$
S(\tau, T)=[\operatorname{HSP}](\tau, T) \times \frac{d D(\tau, T)}{d \tau}
$$

Here, $[\mathrm{HSP}](\tau, T)$ is the HSP produced from HSR mechanism, and $D(\tau, T)$ is the fraction of cells in dead state from three-state cell death model. Both $[\operatorname{HSP}](\tau, T)$ and $D(\tau, T)$ depend on heating temperature and duration time in a nonlinear manner. The total free extracellular HSP concentration $\left([\mathrm{HSP}]_{\mathrm{T}}\right)$ can be calculated by taking the integral of $S(\tau, T)$ over the heating period respective to each temperature from $37^{\circ} \mathrm{C}-45^{\circ} \mathrm{C}$. This equation is expected to help examine the relation between thermally induced HSP expression and cell death and qualitatively predict $[\mathrm{HSP}]_{\mathrm{T}}$ at each temperature and with different exposure times. The incorporation of cell death model with HSR mechanism provides a macro-aspect of how heat-generated dead tumor cells are related to HSPs produced from HSR mechanism; such mechanism provides a micro-description of how the amount of HSP increases due to the presence of denatured $/ \mathrm{misfolded}$ proteins.

\section{Results and discussion Analysis of rate constants}

In the proposed mathematical model, besides the temperature setting, rate constants are also adjustable for the model analysis. Changing rate constants exhibits an increase or a decrease in the fraction of cells in a compartment and alters the HSP synthesis under different degrees of heat stress. Due to the integration of HSR mechanism and three-state death cell model, as shown by Eq. (4), the forward rate constant, $k_{f}$, which corresponds to the dead cell fraction shown in Eq. (2), should relate to the denatured/misfolded protein, which is governed by the rate constant, $k_{11}^{T}$, in the HRS mechanism to some extent. Consequently, the total HSP synthesis, which is hypothetically correlated with the dead cell number, should vary when different $k_{f}$ values are used. As shown in Figure $1 \mathrm{~A}$, four $\overline{k_{f}}$ values, $0.10,0.16,0.20$, and $0.30 \mathrm{~min}^{-1}$ were chosen to demonstrate how changing $k_{f}$ value influences the total HSP production. Although a larger $k_{f}$ value ought to correlate with larger dead cell fraction compared with when a smaller $k_{f}$ value is used, dramatic increase of HSP synthesis was observed when $\overline{k_{f}}=0.16 \mathrm{~min}^{-1}$ was used, while $\overline{k_{f}}=0.20$ or $0.30 \mathrm{~min}^{-1}$ showed nearly unchanged [HSP] under a more enhanced heat stress, indicating that severe cell death at higher temperature does not necessarily give the most HSP synthesis. This phenomenon might result from the fact that the increase of HSPs in response to heat stress takes time to develop, and cells do not have sufficient time to express developed HSP under rapid necrosis. ${ }^{33}$

The backward rate constant, $k_{b}$, in the three-state cell death model ${ }^{32}$ was determined from the experimental results, where each experiment was conducted with a fixed heating time and temperature and was assumed to be invariant with temperature. However, $k_{b}$ describes the biological healing process, as shown in Eq. (2), and therefore is relevant with the thermotolerance effect of HSP. For this reason, $k_{b}$ must have a coupling relation with the HSR mechanism, which includes a temperature-dependent $k_{11}^{T}$, as shown in Eq. (1). Such coupling relation can be achieved by employing Eq. (4), and upon the integration, $k_{b}$ will no longer be temperature independent. Figure 1B shows the results of HSP synthesis at different temperatures with increasing $k_{b}$ values of 0.12 , $0.23,0.47$, and $0.61 \mathrm{~min}^{-1}$. Although a larger $k_{b}$ value results in a decrease in the dead cell fraction, $k_{b}=0.47 \mathrm{~min}^{-1}$ leads 

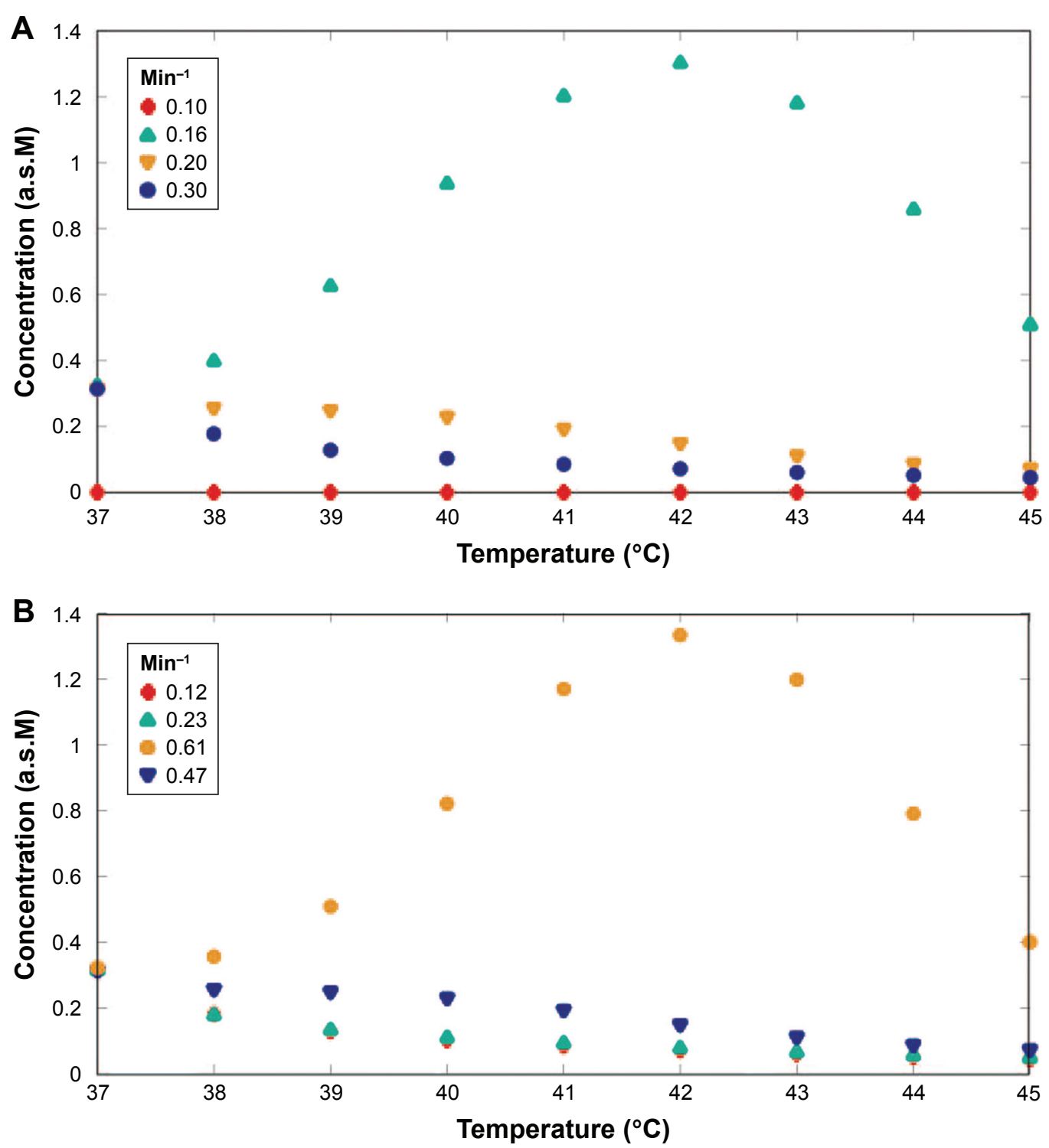

Figure I Total HSPs generated at each temperature, in a.s.M. (A) When $\bar{k}_{f}$ values are adjusted to $0.10,0.16,0.20$, and 0.30 min ${ }^{-1}$. (B) When $k_{b}$ values are adjusted to 0.12 , $0.23,0.47$, and $0.61 \mathrm{~min}^{-1}$. A higher heating temperature, a larger $\bar{k}_{f}$, or a smaller $k_{b}$ do not necessarily give the maximum HSP synthesis, indicating both heating temperature, $T$, and heating duration time, $\tau$, are crucial parameters to achieve optimal immune activation.

Abbreviations: HSPs, heat shock proteins; a.s.M, arbitrary scale molarity.

to a significantly higher production of HSP compared with the other chosen values, which, whether is larger or smaller than $k_{b}=0.47 \mathrm{~min}^{-1}$, shows a slow decrease in $[\mathrm{HSP}]_{\mathrm{T}}$ when heating temperature is increased from $37^{\circ} \mathrm{C}$ to $45^{\circ} \mathrm{C}$, as can been seen in Figure 1B. Owing to the coupled reactions in the HSR mechanism model, constants $k_{f}, k_{b}$, and $k_{11}^{T}$ are all related with each other, making all parameters in the proposed [HSP]-cell death model temperature dependent.

Cell undergoes harsh thermal variations, which might have inverse effect on the activation of adaptive immune responses. It is possible that HSP expression increases when tumor cells struggle to survive under milder heating conditions. ${ }^{33}$ Although production of HSP can be increased by heat to generate antitumor immune responses that retard tumor growth, it requires some time to develop the immune response. ${ }^{33}$ Rate constants analysis in Figure 1 shows that with a specific chosen $k_{f}$ or $k_{b}$ value, synthesis of HSP responds to a gradual increase in temperature, achieving a maximum at a specific temperature, and decreases when temperature is further raised. Such observation indicates that heating exposure time, $\tau$, plays an important role in generating HSP and implies that there is an optimal heating temperature, $\mathrm{T}$, corresponding to the exposure time, $\tau$, to give an optimal HSP synthesis. 


\section{Analysis of optimal HSP synthesis}

To achieve the release of extracellular HSP for immune activation, a critical temperature needs to be exceeded, where it was reported that cytotoxic temperatures for tumor cells are those over $42.5^{\circ} \mathrm{C} .{ }^{34}$ Studies of HSP in the mouse melanoma cell line ${ }^{9}$ showed that the onset of HSP release was at $41^{\circ} \mathrm{C}$, achieved maximum at $43^{\circ} \mathrm{C}$, and the release was completely diminished at $45^{\circ} \mathrm{C}$. Also, it was shown that heating primary tumors at $43^{\circ} \mathrm{C}$ activated antitumor immunity and induced resistance against rechallenge. ${ }^{13}$ With respect to these in vitro results, the proposed [HSP] cell death model was analyzed to see if the simulated results agree with the experimental data. Figure 2 shows that, by adjusting either $\overline{k_{f}}$ to $0.15 \mathrm{~min}^{-1}$ or $k_{b}$ to $0.62 \mathrm{~min}^{-1}$, maximum HSP synthesis can be achieved at $43^{\circ} \mathrm{C}$. The initiation of HSP synthesis was under milder thermal stress $\left(<43^{\circ} \mathrm{C}\right)$, which is resulted from the thermotolerance effect present in the weakest cell systems due to intracellular HSPs. These intracellular HSPs do not contribute to the activated antitumor immunity because the temperature for tumor cell cytotoxicity is not yet reached. HSPs heated at a temperature above $42.5^{\circ} \mathrm{C}$ will be released from the dead tumor cells, travel to and enter the extracellular milieu, recognize the antigens released from the cytotoxic tumor cells, and activate the antitumor immunity. It can be seen in Figure 2 that a maximum HSP synthesis is achieved at the cytotoxic temperature of $43^{\circ} \mathrm{C}$, followed by a gradual decrease when the temperature continuously increases. This result again suggests that extracellular HSP synthesis does not respond linearly to the increase of temperature. It agrees with our previous argument that a milder cytotoxic heating temperature could be more stimulatory than a higher one. A milder temperature that is sufficient to cause tumor cell necrosis can help prevent overheating the surrounding tissues if an accurate prediction of the tissue response cannot be achieved. It has to be noted that, in combination with a more focusing and localized hyperthermia treatment, an even more milder temperature $\left(\leq 42.5^{\circ} \mathrm{C}\right)$ could potentially achieve the same efficiency of antitumor immunity. ${ }^{35} \mathrm{In}$ our particular interest of developing the model for immune activation at cytotoxic temperatures but not the immunomodulatory activities under fever-like hyperthermia exposure such as lymphocyte function, ${ }^{36}$ it is more feasible to use this model for localized hyperthermia treatment than a wholebody hyperthermia.

\section{Effect of heating duration time on HSP synthesis}

The results in Figures 1 and 2 point out the importance of heating duration time, $\tau$, in synthesizing HSP. Duration of heating might induce clustering in the plasma membrane and changes in membrane fluidity, which could affect the induced immunity ${ }^{37}$ Studies have shown that heating tumors at $43^{\circ} \mathrm{C}$ for 30 min expressed the largest amount of HSP, activated antitumor immunity, and led to a reduction in metastases. ${ }^{9,13}$ Consequently, to achieve the optimal antitumor immune activation, heating duration time is a crucial parameter to analyze. Table 1 shows the optimized parameter values, while in Figure $3 \mathrm{~A}$ the optimized results are shown in terms

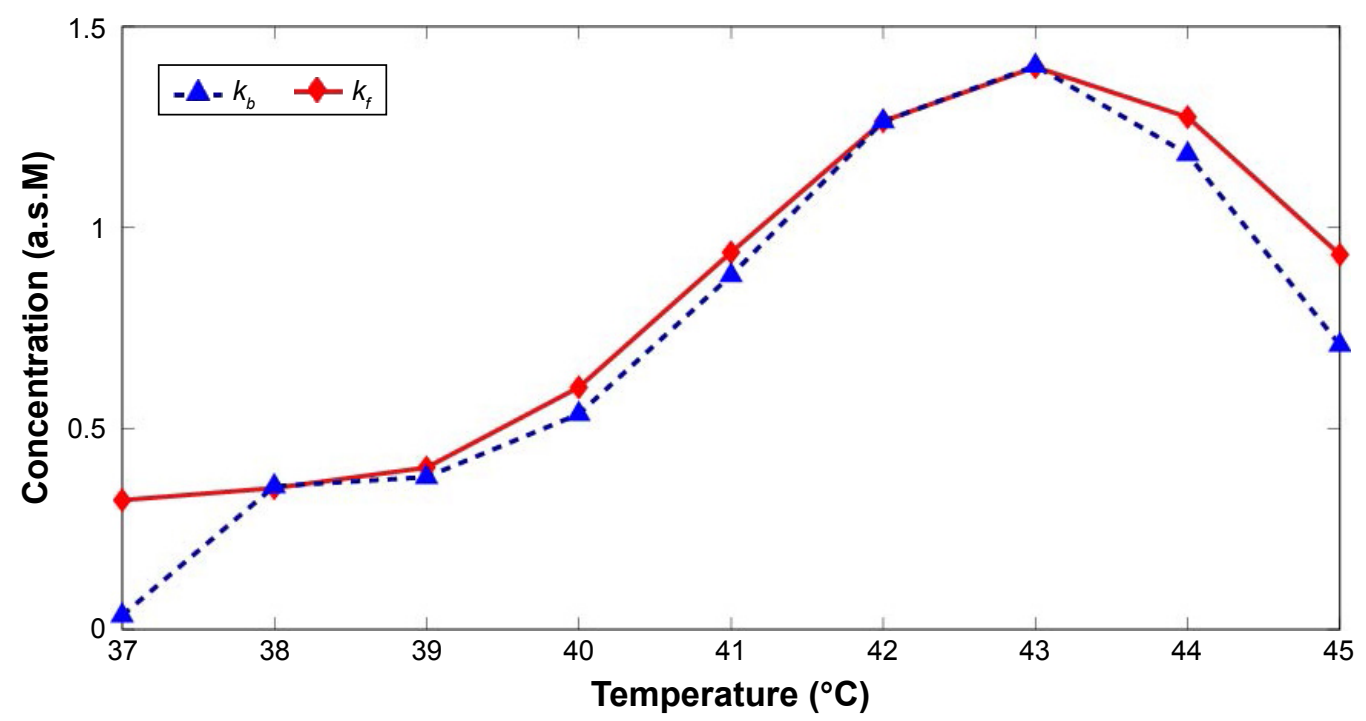

Figure 2 Total HSPs generated at each temperature. Comparison between the chosen $k_{f}$ and $k_{b}$ values. After each rate constant adjustment, a maximum HSP synthesis at $43^{\circ} \mathrm{C}$ can be achieved and HSP amount decreases, if the temperature is further raised. $\bar{k}_{f}=0.15$ and $k_{b}=0.62 \mathrm{~min}^{-1}$.

Abbreviations: HSPs, heat shock proteins; a.s.M, arbitrary scale molarity. 
Table I List of [HSP]-cell death model parameters and values for optimized results

\begin{tabular}{ll}
\hline Parameter & Optimized value \\
\hline$\overline{k_{f}}$ & $0.15 \mathrm{~min}^{-1}$ \\
$k_{b}$ & $0.62 \mathrm{~min}^{-1}$ \\
$m_{11}$ & 0.015 \\
$T_{k}$ & $40.5^{\circ} \mathrm{C}$ \\
\hline
\end{tabular}

Abbreviation: HSP, heat shock protein.

of HSP generation rate and accumulated HSP against heating duration time. Using either the optimized $\overline{k_{f}}$ or $k_{b}$ value, a maximum HSP synthesis at $43^{\circ} \mathrm{C}$ was achieved. Nevertheless, despite using the optimized $\overline{k_{f}}$ value, dead cell fraction still increases when temperature is raised from $38^{\circ} \mathrm{C}$ to $45^{\circ} \mathrm{C}$ (Figure 4), indicating that the production of HSP is not linearly related with dead cell number. This observation also implies that it might not be necessary to completely diminish the tumor cells to activate HSP-induced antitumor immunity. Although HSPs were synthesized at a slightly faster rate when a higher temperature $\left(44^{\circ} \mathrm{C}\right.$ or $\left.45^{\circ} \mathrm{C}\right)$ was applied, as shown in Figure $3 \mathrm{~A}$, a higher [HSP] was generated after heating at $43^{\circ} \mathrm{C}$ for approximately $100 \mathrm{~min}$. Comparably, assuming under the same condition, HSPs were generated at a much slower rate (and also less amount were obtained) when using heating temperatures that are close to body temperature. Figure $3 \mathrm{~B}$ shows the optimized $k_{b}$ result in terms of accumulated HSP against heating time. Agreeably,

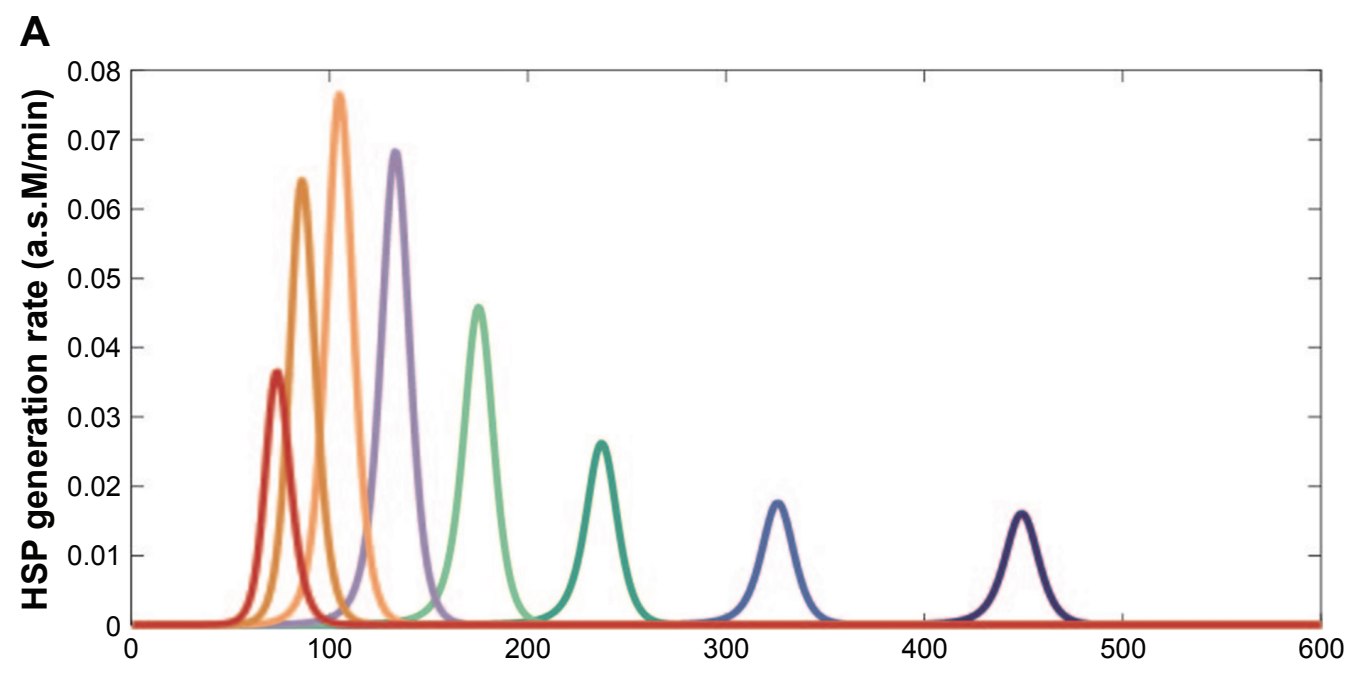

B

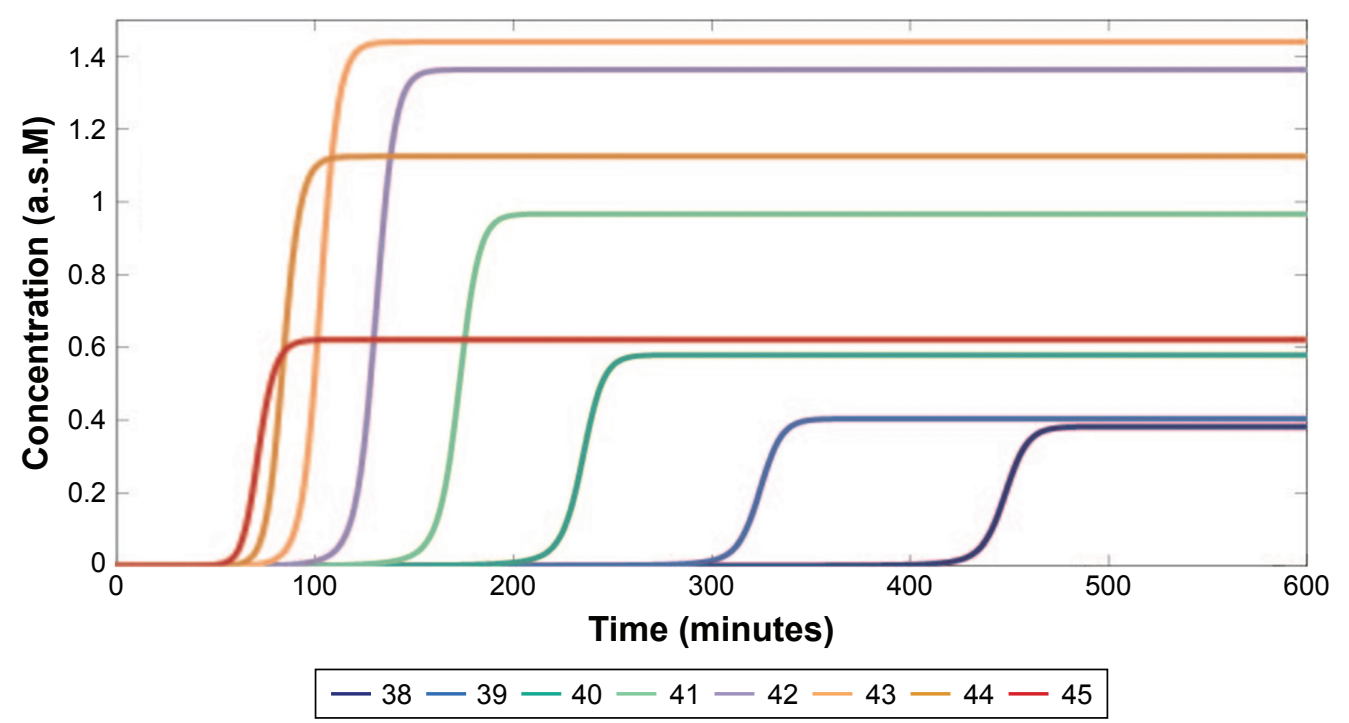

Figure 3 Maximum HSP synthesis is achieved at $43^{\circ} \mathrm{C}$ by using either the optimized $k_{f}$ or $k_{b}$. Results for cytotoxic temperatures ( $\left.\geq 43^{\circ} \mathrm{C}\right)$ are significantly different from those that are around the body temperature. (A) HSP generation rate, $S(\tau, T)$, for different heating temperatures using the optimized $k_{b}$ value. a.s.M/min. (B) Heating duration time and the accumulated HSP at different temperatures using the optimized $k_{b}$ value. Abbreviations: HSPs, heat shock proteins; a.s.M, arbitrary scale molarity. 


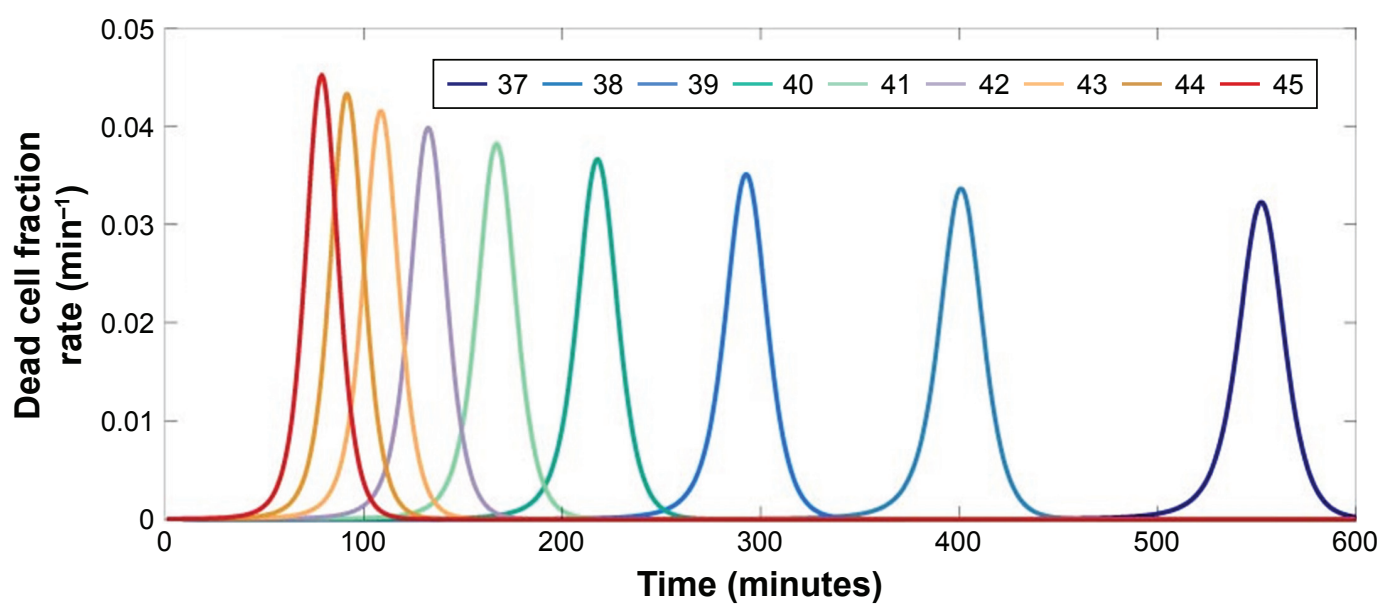

Figure 4 Dead cell fraction rate, $\frac{d D(\tau, T)}{d \tau}$, generated by cell death model using the optimized $\overline{k_{f}}$ value. Even though maximum HSP was obtained at $43^{\circ} \mathrm{C}$, when heating temperature was raised, cells died at a faster rate and the final dead cell fraction increased along with the applied temperatures. Abbreviation: HSP, heat shock protein.

HSP accumulation at temperatures $44^{\circ} \mathrm{C}$ and $45^{\circ} \mathrm{C}$, although accumulated faster when the initial heating is applied, leveled out before HSP synthesis at temperatures $43^{\circ} \mathrm{C}$ achieved its maximum (slightly $>100 \mathrm{~min}$ ), with a lower final [HSP]. Similar result was obtained when using the optimized $k_{f}$ value (Figure 5). The heating duration is defined as the time required for each specific temperature to reach its final accumulated [HSP]. It can be seen that even though the difference of heating duration between the cytotoxic temperatures, $43^{\circ} \mathrm{C}, 44^{\circ} \mathrm{C}$, and $45^{\circ} \mathrm{C}$, is small, the final HSP accumulation at $43^{\circ} \mathrm{C}$ is significantly higher than the other two temperatures.
It shows that a relatively milder cytotoxic temperature can achieve a much desired HSP production. By comparing with the HSP synthesis performed at $43^{\circ} \mathrm{C}$, a lower temperature $\left(38^{\circ} \mathrm{C}\right)$ only produces approximately 4 -fold less amount of $[\mathrm{HSP}]_{\mathrm{T}}$ with a much longer heating duration (about 6-fold longer), as can be seen in Figure 3B.

Figure 6 shows the optimal HSP synthesis with respect to duration time, $\tau$, and heating temperature, $T$, ranged from $37^{\circ} \mathrm{C}$ to $45^{\circ} \mathrm{C}$. It can be seen that to achieve a maximum HSP synthesis at $43^{\circ} \mathrm{C}, \sim 100 \mathrm{~min}$ of duration time is required. Such an increase in HSP synthesis is not visible for other heating

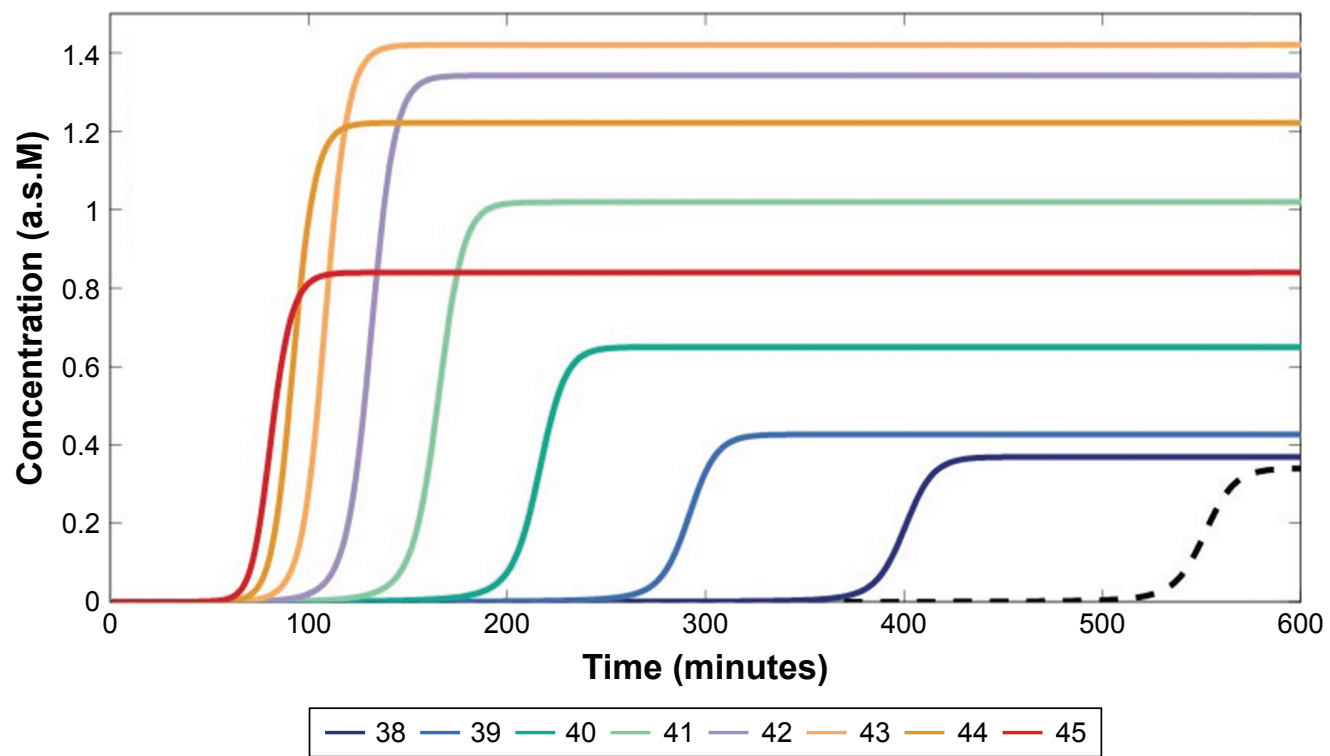

Figure 5 Heating duration time, $\tau$, and the accumulated HSP at different temperatures, $T$, using the optimized $\overline{k_{f}}$ value. A maximum final accumulated HSP was achieved at $43^{\circ} \mathrm{C}$ despite an enhanced HSP generation at higher temperatures when heat was applied initially. The dashed line is for the result obtained at body temperature $37^{\circ} \mathrm{C}$, as a reference.

Abbreviations: HSP, heat shock protein; a.s.M, arbitrary scale molarity. 


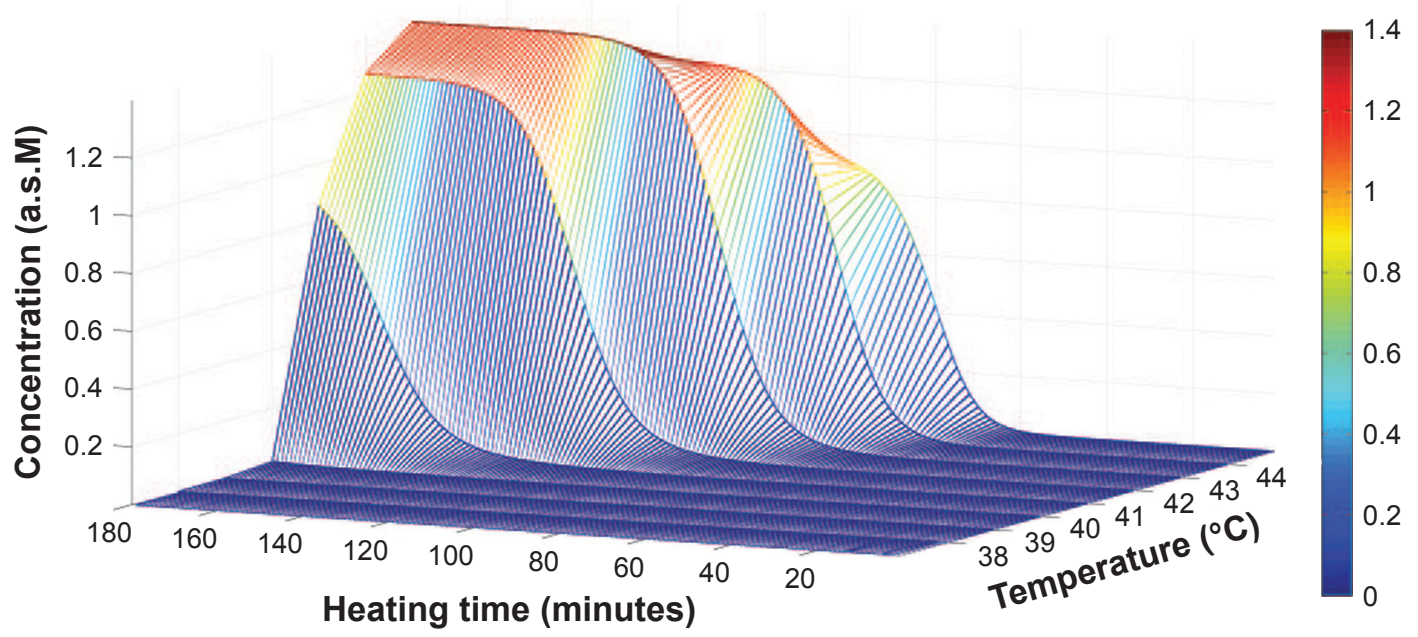

Figure 6 Maximum HSP synthesis with respect to heating temperature, $T$, and duration time, $\tau$, using the optimized $\overline{k_{f}}$ value. Duration time is approximately I00 min to achieve maximum HSP synthesis at $43^{\circ} \mathrm{C}$. Maximum amount of HSP generated at the other temperatures is smaller than the amount achieved at $43^{\circ} \mathrm{C}$, even a longer heating time is applied. Abbreviations: HSP, heat shock protein; a.s.M, arbitrary scale molarity.

temperatures, even if a longer duration time is applied. The calculated optimal heating duration time (100 $\mathrm{min})$ is longer compared to that in the previous in vitro studies. ${ }^{9,13}$ It is crucial to include the biological factors that might modify the response because in vitro studies may not accurately reflect the cellular response in a physiologically relevant in vivo environment. ${ }^{29}$ In vitro experiments are not able to include all the real-time regulation of HSP synthesis, whereas the HSR mechanism model used in this study consists of a system of 12 nonlinear ordinary differential equations (ODEs) describing the evolution of the key variables in the HSP synthesis regulation with respect to temperature. Sensitivity to hyperthermia varies among different kinds of cancer cells, ${ }^{4}$ and the heating efficiency also depends on how temperature is administere to the cells. ${ }^{29}$ Moreover, HSPs might secrete into extracellular space through a possible pathway. ${ }^{23}$ As a result, it is necessary to put factors such as parameter dependency upon different cell lines, cell incubation times, and heat administrating methods into consideration to achieve an optimal extracellular HSP synthesis.

\section{Conclusion}

Traditional cancer treatments normally focus on reducing tumor burden, while stimulation of systemic self-defense against metastatic tumors has not been taken into much consideration. ${ }^{6}$ Due to intracellular HSP's role of cell-protective chaperons, they tend to protect cancer cells that undergo heat stress. As a result, previous studies often focus on minimizing HSP expression in the targeted tumor region. ${ }^{11}$ In our previous work, we developed magnetic resonance nanotheranostic hyperthermia using nontoxic, biocompatible magnetic nanoparticles to target and accumulate at the lesions to generate enhanced contrast for early lesion detection and generate heat to kill lesion cells directly through hyperthermia or indirectly through thermal activation and control releasing of drugs. ${ }^{38-43}$ In this study, instead of focusing on producing a direct cell-killing effect, hyperthermia treatment serves to induce the antitumor immune system activated by the extracellular HSPs released from the heat-treated necrotic tumor cells. Such immunity is expected to detect and attack all metastases. We incorporate cell death model with extracellular HSP expression to investigate HSP synthesis under various temperatures and exposure times.

The model produced a maximum extracellular HSP synthesis at $43^{\circ} \mathrm{C}$ with an approximate exposure time of $100 \mathrm{~min}$, which is in agreement with the significant cell death obtained when cells or tissues are heated up to temperatures of $\geq 42^{\circ} \mathrm{C}$ for 1 or more hours. Discordant results of exposure time required to achieve a maximum extracellular HSP synthesis at $43^{\circ} \mathrm{C}$ between the proposed model and in vitro data may be due to the possible effects of different cell lines ${ }^{4,32}$ or a possible secretory pathway into extracellular space for HSPs. ${ }^{23}$ The maximum amount achieved at $43^{\circ} \mathrm{C}$ was then followed by a decrease in HSP synthesis when the temperature was further raised, suggesting that extracellular HSP synthesis does not respond linearly to the increase in temperature. Such outcome confirms that a milder cytotoxic heating temperature could be more stimulatory than a higher one. In conclusion, our model is capable of predicting the optimal temperature and exposure time to generate HSPs involved in the antitumor immune activation, with a goal to promote tumor regression and reduce metastasis. 


\section{Acknowledgments}

This work was supported by the Camille and Henry Dreyfus Foundation (TC-05-053), National Science Foundation (CHE-1112574 and CHE-1416598), University of California Cancer Research Award (CRR-13-201412), and Hirshberg Foundation for Pancreatic Cancer Research.

\section{Disclosure}

The authors report no conflicts of interest in this work.

\section{References}

1. Huang HC, Yang Y, Nanda A, Koria P, Rege K. Synergistic administration of photothermal therapy and chemotherapy to cancer cells using polypeptide-based degradable plasmonic matrices. Nanomedicine (Lond). 2011;6(3):459-473.

2. Bañobre-López M, Teijeiro A, Rivas J. Magnetic nanoparticle-based hyperthermia for cancer treatment. Rep Pract Oncol Radiother. 2013; 18(6):397-400.

3. Huang HS, Hainfeld JF. Intravenous magnetic nanoparticle cancer hyperthermia. Int J Nanomedicine. 2013;8:2521-2532.

4. Stapf M, Pömpner N, Teichgräber U, Hilger I. Heterogeneous response of different tumor cell lines to methotrexate-coupled nanoparticles in presence of hyperthermia. Int J Nanomedicine. 2016;11:485-500.

5. Chu KF, Dupuy DE. Thermal ablation of tumours: biological mechanisms and advances in therapy. Nat Rev Cancer. 2014;14(3):199-208.

6. Zhu J, Zhang Y, Zhang A, He K, Liu P, Xu LX. Cryo-thermal therapy elicits potent anti-tumor immunity by inducing extracellular Hsp70dependent MDSC differentiation. Sci Rep. 2016;6(1):27136.

7. Haen SP, Gouttefangeas C, Schmidt D, et al. Elevated serum levels of heat shock protein 70 can be detected after radiofrequency ablation. Cell Stress Chaperones. 2011;16(5):495-504.

8. Seigneuric R, Mjahed H, Gobbo J, et al. Heat shock proteins as danger signals for cancer detection. Front Oncol. 2011;1:1-10.

9. Ito A, Fujioka M, Tanaka K, Kobayashi T, Honda H. Screening of cytokines to enhance vaccine effects of heat shock protein 70-rich tumor cell lysate. J Biosci Bioeng. 2005;100(1):36-42.

10. Tanaka K, Ito A, Kobayashi T, et al. Intratumoral injection of immature dendritic cells enhances antitumor effect of hyperthermia using magnetic nanoparticles. Int J Cancer. 2005;116(4):624-633.

11. Frey B, Weiss EM, Rubner Y, et al. Old and new facts about hyperthermiainduced modulations of the immune system. Int J Hyperth. 2012; 28(6):528-542.

12. Todryk S, Melcher AA, Hardwick N, et al. Heat shock protein 70 induced during tumor cell killing induces Th1 cytokines and targets immature dendritic cell precursors to enhance antigen uptake. J Immunol. 1999;163(3):1398-1408.

13. Toraya-Brown S, Sheen MR, Zhang P, et al. Local hyperthermia treatment of tumors induces $\mathrm{CD} 8+\mathrm{T}$ cell-mediated resistance against distal and secondary tumors. Nanomedicine. 2014;10(6):1273-1285.

14. McNulty S, Colaco CA, Blandford LE, Bailey CR, Baschieri S, Todryk S. Heat-shock proteins as dendritic cell-targeting vaccines getting warmer. Immunology. 2013;139(4):407-415.

15. Shevtsov MA, Yakovleva LY, Nikolaev BP, et al. Tumor targeting using magnetic nanoparticle Hsp70 conjugate in a model of C6 glioma. Neuro Oncol. 2014;16(1):38-49.

16. Shevtsov MA, Nikolaev BP, Yakovleva LY, et al. 70-kDa heat shock protein coated magnetic nanocarriers as a nanovaccine for induction of anti-tumor immune response in experimental glioma. J Control Release. 2015;220(Pt A):329-340.

17. Rylander MN, Diller KR, Wang S, Aggarwal SJ. Correlation of HSP70 expression and cell viability following thermal stimulation of bovine aortic endothelial cells. $J$ Biomech Eng. 2005;127(5):751-757.
18. Rylander MN, Feng Y, Bass J, Diller KR. Thermally induced injury and heat-shock protein expression in cells and tissues. Ann N Y Acad Sci. 2005;1066:222-242.

19. Samali A, Holmberg CI, Sistonen L, Orrenius S. Thermotolerance and cell death are distinct cellular responses to stress: dependence on heat shock proteins. FEBS Lett. 1999;461(3):306-310.

20. Hildebrandt $\mathrm{B}, \mathrm{Wust} \mathrm{P}, \mathrm{Ahlers} \mathrm{O}$, et al. The cellular and molecular basis of hyperthermia. Crit Rev Oncol Hematol. 2002;43(1):33-56.

21. Lepock JR. Cellular effects of hyperthermia: relevance to the minimum dose for thermal damage. Int J Hyperth. 2003;19(3):252-266.

22. Dewhirst MW, Vujaskovic Z, Jones E, Thrall D. Re-setting the biologic rationale for thermal therapy. Int J Hyperth. 2005;21(8):779-790.

23. Vega VL, Rodriguez-Silva M, Frey T, et al. Hsp70 translocates into the plasma membrane after stress and is released into the extracellular environment in a membrane-associated form that activates macrophages. J Immunol. 2008;180(6):4299-4307.

24. Rybinski M, Szymanska Z, Lasota S, Gambin A. Modelling the efficacy of hyperthermia treatment. J Roy Soci Inter. 2013;10(88):20130527.

25. Szymańska Z, Zylicz M. Mathematical modeling of heat shock protein synthesis in response to temperature change. $J$ Theor Biol. 2009;259(3):562-569.

26. Peper A, Grimbergen CA, Spaan JA, Souren JE, van Wijk R. A mathematical model of the hsp70 regulation in the cell. Int J Hyperthermia. 1998;14(1):97-124.

27. Lepock JR, Frey HE, Ritchie KP. Protein denaturation in intact hepatocytes and isolated cellular organelles during heat shock. $J$ Cell Biol. 1993;122(6):1267-1276.

28. Hall DM, Oberley TD, Moseley PM, et al. Caloric restriction improves thermotolerance and reduces hyperthermia-induced cellular damage in old rats. FASEB J. 2000;14(1):78-86.

29. Song AS, Najjar AM, Diller KR. Thermally induced apoptosis, necrosis, and heat shock protein expression in 3D culture. $J$ Biomech Eng. 2014;136(7):71006

30. Kregel KC. Invited Review: Heat shock proteins: modifying factors in physiological stress responses and acquired thermotolerance. $J$ Appl Physiol. 2002;92(5):2177-2186.

31. Shevtsov M, Huile G, Multhoff G. Membrane heat shock protein 70: a theranostic target for cancer therapy. Philos Trans R Soc Lond B Biol Sci. 2018;373(1738):20160526.

32. O'Neill DP, Peng T, Stiegler P, et al. A three-state mathematical model of hyperthermic cell death. Ann Biomed Eng. 2011;39(1): $570-579$.

33. Toraya-Brown S, Fiering S. Local tumour hyperthermia as immunotherapy for metastatic cancer. Int J Hyperth. 2014;30(8):531-539.

34. Dewey WC, Hopwood LE, Sapareto SA, Gerweck LE. Cellular responses to combinations of hyperthermia and radiation. Radiology. 1977;123(2):463-474.

35. Tsang YW, Huang CC, Yang KL, et al. Improving immunological tumor microenvironment using electro-hyperthermia followed by dendritic cell immunotherapy. BMC Cancer. 2015;15(1):708.

36. Di YP, Repasky EA, Subjeck JR. Distribution of HSP70, protein kinase $\mathrm{C}$, and spectrin is altered in lymphocytes during a fever-like hyperthermia exposure. J Cell Physiol. 1997;172(1):44-54.

37. Mace TA, Zhong L, Kilpatrick C, et al. Differentiation of CD $8+\mathrm{T}$ cells into effector cells is enhanced by physiological range hyperthermia. J Leukoc Biol. 2011;90(5):951-962.

38. Chen KJ, Wolahan SM, Wang H, et al. A small MRI contrast agent library of gadolinium(III)-encapsulated supramolecular nanoparticles for improved relaxivity and sensitivity. Biomaterials. 2011;32(8): 2160-2165.

39. Ho LC, Hsu CH, Ou CM, et al. Unibody core-shell smart polymer as a theranostic nanoparticle for drug delivery and MR imaging. Biomaterials. 2015;37:436-446.

40. Li Z, Hsu CH, Dimitrov N, et al. Sensitive imaging of magnetic nanoparticles for cancer detection by active feedback magnetic resonance. Magn Reson Med. 2015;74(1):33-41. 
41. Yao J, Hsu CH, Li Z, et al. Magnetic resonance nano-theranostics for glioblastoma multiforme. Curr Pharm Des. 2015;21(36):5256-5266.

42. Wang C, Li Z, Lin YY. Optimizing magnetic nanoparticle hyperthermia effect in MR nanomedicine. Chin J Magn Reson. 2015;32(2):248-260.
43. Wang $\mathrm{C}$, Hsu CH, Li Z, et al. Effective heating of magnetic nanoparticle aggregates for in vivo nano-theranostic hyperthermia. Int $J$ Nanomedicine. 2017;12:6273-6287.

\section{Publish your work in this journal}

The International Journal of Nanomedicine is an international, peerreviewed journal focusing on the application of nanotechnology in diagnostics, therapeutics, and drug delivery systems throughout the biomedical field. This journal is indexed on PubMed Central, MedLine, CAS, SciSearch $\AA$, Current Contents ${ }^{\circledR} /$ Clinical Medicine,
Journal Citation Reports/Science Edition, EMBase, Scopus and the Elsevier Bibliographic databases. The manuscript management system is completely online and includes a very quick and fair peer-review system, which is all easy to use. Visit http://www.dovepress.com/ testimonials.php to read real quotes from published authors.

Submit your manuscript here: http://www.dovepress.com/international-journal-of-nanomedicine-journal 